\title{
In vitro retention of a new thermoplastic titratable
}

\section{mandibular advancement device [version 1; peer review: 2}

\section{approved]}

\author{
Marc Braem ${ }^{1-3}$ \\ ${ }^{1}$ Lab Dental Materials, University of Antwerp, Antwerp, 2610, Belgium \\ ${ }^{2}$ Special Care Dentistry, University Hospital Antwerp, Edegem, 2650, Belgium \\ ${ }^{3}$ Faculty of Medicine and Health Sciences, University of Antwerp, Antwerp, 2610, Belgium
}

V1 First published: 26 Feb 2015, 4:56

https://doi.org/10.12688/f1000research.6061.1

Latest published: 26 Feb 2015, 4:56

https://doi.org/10.12688/f1000research.6061.1

\section{Abstract}

Oral appliance (OA) therapy with a mandibular advancement device $(\mathrm{OAm})$ is a non-invasive, alternative approach to maintaining upper airway patency. The main requirement for an OAm to be effective is the adequate retention on the teeth while the patient is asleep. We evaluated the retentive forces of a new low-cost, customizable, titratable, thermoplastic OAm (BluePro ${ }^{\circledR}$; BlueSom, France). Dental impressions and casts were made for one patient with complete upper and lower dental arches including the third molars and class II bite proportions. A setup based on Frasaco ANA-4 models was also used. Two protrusive positions of the mandible were investigated: 3 $\mathrm{mm}$ and $8 \mathrm{~mm}$, representing respectively $25 \%$ and $65 \%$ of the maximal protrusion. The forces required to remove the BluePro ${ }^{\circledR}$ device from the carriers were recorded continuously over 730 cycles (=365 days, twice a day) to simulate 1 year of clinical use. At $8 \mathrm{~mm}$ protrusion the BluePro ${ }^{\circledR}$ device showed retentive forces of $\sim 27 \mathrm{~N}$. There was a slight but non-significant decrease in retentive forces in the tests on the epoxified carriers which was not found on the ANA-4 carriers. There were no significant differences between the carriers as a function of protrusion. The BluePro ${ }^{\circledR}$ device tested in the present study possesses sufficient retention forces to resist initial jaw opening forces and full mouth opening forces estimated to be $\sim 20 \mathrm{~N}$. It could therefore broaden the indications for use of thermoplastic OAms. It could provide a temporary OAm while a custom-made OAm is being manufactured or repaired. Patients could be provided with a low-cost try-out device capable of reliable titration, providing an indication of effectiveness and of patient acceptance of an OAm, although the effect of device shape and size on therapeutic outcome is not yet known. Finally it could provide an affordable OAm solution in resource-restricted healthcare settings.

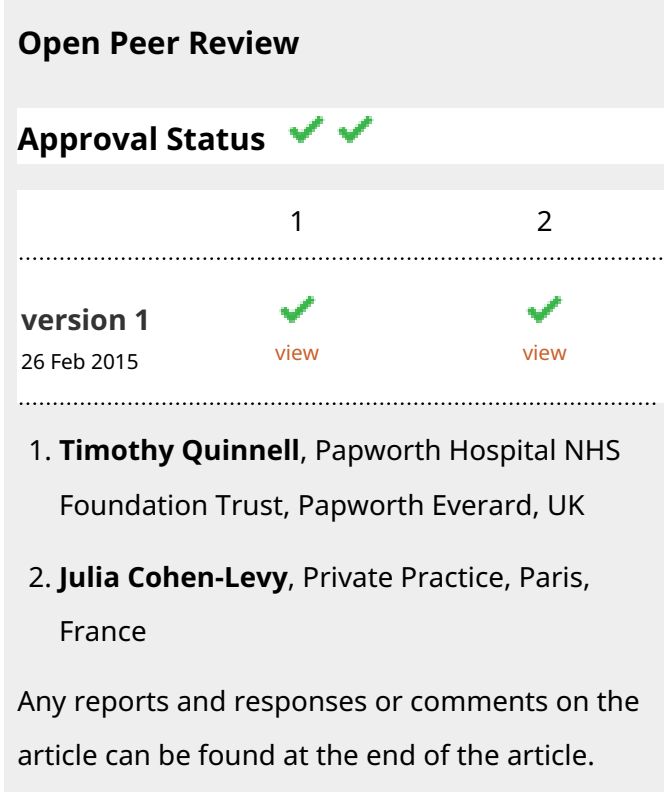




\section{Keywords}

mandibular advancement device, obstructive sleep apnoea , oral appliance, retention force

Corresponding author: Marc Braem (marc.braem@uantwerpen.be)

Competing interests: The author is the promotor of a SomnoMed Research Grant. The author has no competing interest relating to BlueSom.

Grant information: BlueSom France provided funding for this project including the supply of BluePro® devices free of charge and payment for final manuscript editing by Newmed Publishing Services.

Copyright: @ 2015 Braem M. This is an open access article distributed under the terms of the Creative Commons Attribution License, which permits unrestricted use, distribution, and reproduction in any medium, provided the original work is properly cited. Data associated with the article are available under the terms of the Creative Commons Zero "No rights reserved" data waiver (CCO 1.0 Public domain dedication).

How to cite this article: Braem M. In vitro retention of a new thermoplastic titratable mandibular advancement device [version 1; peer review: 2 approved] F1000Research 2015, 4:56 https://doi.org/10.12688/f1000research.6061.1

First published: 26 Feb 2015, 4:56 https://doi.org/10.12688/f1000research.6061.1 


\section{Introduction}

Oral appliance (OA) therapy with mandibular advancement devices $(\mathrm{OAm})$ is becoming the main non-invasive and alternative approach to continuous positive airway pressure. By advancing the mandible and tongue, upper airway patency is favoured during sleep thereby preventing upper airway collapse ${ }^{1}$. Although many types of OAm are available ${ }^{2-5}$, each requires retention on the teeth to maintain protrusion of the mandible during sleep. Lack of retention causes loosening of the OAm and may result in reduced efficacy of treatment, patient complaints about poor fit and an increased risk of side effects ${ }^{4,6,7}$.

The main disadvantages of custom-made OAm are not only the cost, but also, more importantly, the time required to manufacture the device, while the obstructive sleep apnoea (OSA)-patient is not being treated. A comparable situation arises when the OAm of a patient under treatment needs to be repaired in the dental laboratory. Furthermore, clinical practice demonstrates that patients are often not willing to leave the OAm for repair because they have become dependent on the device for a good night's sleep.

The primary requirement for an OAm to be effective is the adequate retention on the teeth while the patient is asleep. Recently, in vitro testing of such retentive characteristics of different types of OAm, including thermoplastic ones, was reported in the literature ${ }^{8}$, demonstrating that generalization of the retentive characteristics with respect to the design and type of an OAm is not pertinent.

The present report compares the retentive forces of a novel customizable titratable thermoplastic low-cost OAm to other devices reported previously in the literature.

\section{Materials and methods}

Supporting tooth arcs

An experimental customizable titratable thermoplastic oral appliance commercialized for use by healthcare professionals under the name BluePro ${ }^{\circledR}$, was tested (BlueSom, France) (Figure 1).

Dental impressions and casts were made (Impregum Penta Soft Medium, 3M ESPE AG, Germany) for one patient with complete upper and lower dental arches including the third molars and

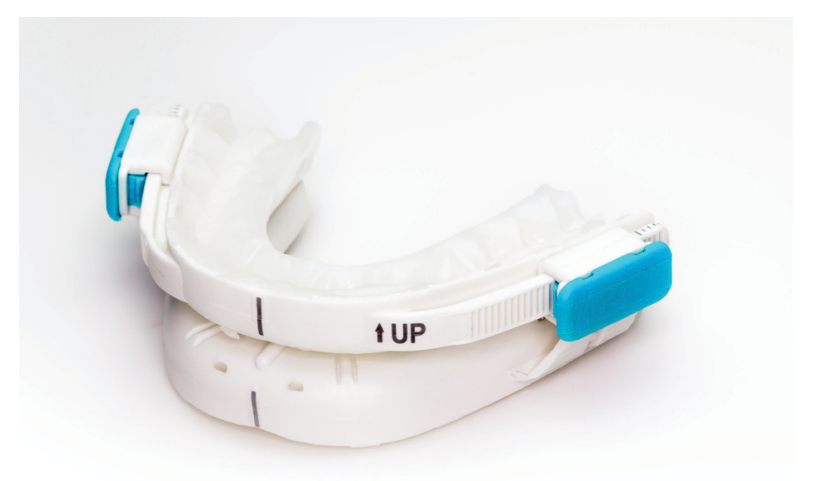

Figure 1. BluePro ${ }^{\circledR}$ device.
Class II bite proportions. A setup based on Frasaco ANA-4 models (Frasaco, Germany) was also made.

The protrusion to be incorporated into the BluePro ${ }^{\circledR}$ was measured from the vestibular rim of the incisive edge of the upper left central incisor, in a horizontal plane up to the lingual rim of the incisive edge of the lower left central incisor. These distances represent clinically relevant protrusive positions as noted during actual OA therapy in patients. Two protrusive positions were investigated in the present tests: $3 \mathrm{~mm}$ and $8 \mathrm{~mm}$ of protrusion, representing $25 \%$ and $65 \%$ of the maximal protrusion in the particular patient.

\section{Sample production}

Samples of the BluePro ${ }^{\circledR}$ were fitted onto the epoxified casts $(n=10$ for each protrusion) as well as on the ANA-4 models ( $n=10$ only for $8 \mathrm{~mm}$ protrusion, see further) according to the manufacturer's instructions (BluePro ${ }^{\circledR}$ user leaflet). The exact procedure was as follows:

Boiling water was poured into a flat-bottomed polypropylene box. The upper and lower parts of the BluePro ${ }^{\circledR}$ were then placed into the hot water until the inner material in both parts became fully transparent. A timer was started and the upper part was taken out of the water and put to one side to cool down for 30s. The same procedure was then repeated for the lower part and the upper part was immediately shaped onto the upper carrier, either the epoxified cast or ANA-4 carrier, depending on the test required.

The carrier was pressed firmly into the upper part of the BluePro ${ }^{\circledR}$, sliding it a bit forward to create an optimal fit into the tray of the BluePro ${ }^{\circledR}$. Finger pressure was then applied and the softened material on the inside of the tray was immediately flattened. The softened material was also pressed into shape at the outside of the tray to fit it tightly around the carrier. Both the carrier and the BluePro ${ }^{\circledR}$ were put on a flat table, the BluePro ${ }^{\circledR}$ facing down, and a dead weight was placed on top to simulate gentle biting and to immobilize the device in the required position, without popping or creeping out. This procedure was completed within 30 s, then was repeated for the lower jaw.

Both parts were allowed to cool down for $5 \mathrm{~min}$. The thermoplastic material inside the tray was then checked to see if it had become cold and opaque again and the BluePro ${ }^{\circledR}$ was removed from the carrier and rinsed in cold water. If necessary, sharp edges of superfluous softened material were removed, as well as any material that had crept into the rails or locks. The device was then dried and stored in the original packing at room temperature prior to testing.

\section{Sample mounting}

The casts and ANA-4 models were mounted in average value inclination of the occlusal plane and centred in a hydraulic cyclic test machine (Dartec HC10, Testbench Dartec 9600 Controller; Dartec, UK) as described previously ${ }^{8}$.

When mounting the casts, stubs were added to these to facilitate removal and reinsertion. Both upper and lower casts were mounted at a fixed position, giving a protrusion of $3 \mathrm{~mm}$ or $8 \mathrm{~mm}$, depending on the test. 
When mounting the ANA-4 carriers into the fatigue machine, first a bite-registration was made with silicone on a plastic spoon with a protrusion of $8 \mathrm{~mm}$ compared to the habitual occlusion. Using this bite registration as a reference, the ANA-4 carriers were fixed in a reproducible way so that all $\mathrm{BluePro}^{\circledR}$ samples were tested in an identical protrusion. Due to technical limitations of this version regarding the length of the rim for the clasps, it turned out that the $3 \mathrm{~mm}$ protrusion could not be tested. Next, for the fitting of the OAm-TP on the ANA-4 carriers a specific procedure was followed.

First the upper and lower parts of the BluePro ${ }^{\circledR}$ were inserted onto the carriers but without interconnecting (Figure 2A). The upper carrier together with the OAm could now move up and down freely as the piston moved. The lower model stayed fixed to the bottom of the machine. The upper carrier with the BluePro ${ }^{\circledR}$ was then lowered until it touched the lower carrier establishing the zero-position calibration for the stroke. Next, both the upper and lower parts of the OAm were connected by sliding the locks onto the rails, but without locking and firmly seated thereafter by finger pressure. The locks were then closed and the BluePro ${ }^{\circledR}$ was positioned correctly in the machine for the given protrusion (Figure 2B). In this position, there appeared to be a gap in the frontal region preventing the correct repositioning at reinsertion during the fatigue test. To avoid this phenomenon, a passive splint in silicone putty was inserted (Figure 2C). The upper piston was moved upwards until the BluePro ${ }^{\circledR}$ came loose from either of the ANA-4 carriers and with the sample now hanging free and unloaded, the load (force) was set to zero so that both the stroke (displacement) and load (force) were calibrated correctly. The fatigue test was then started.

\section{Sample measurements}

The forces required to remove the BluePro ${ }^{\circledR}$ from the carriers were continuously recorded during 730 cycles ( $=365$ days, twice a day) at $35^{\circ} \mathrm{C}$ in a dry environment to simulate 1 year of clinical use. Data were stored in CSV format for export to Excel (Microsoft, USA, see Dataset 1 and Figshare Dataset).

The test was setup as a stroke-controlled test with a triangular waveform, thus the piston repeatedly moved up and down with a given stroke and frequency. The piston speed was $7.5 \mathrm{~mm} / \mathrm{s}$ and was kept constant during all tests. The stroke length was set to $7.5 \mathrm{~mm}$, back and forth from $0.0 \mathrm{~mm}$ to $7.5 \mathrm{~mm}$. This stroke was chosen carefully so that the BluePro ${ }^{\circledR}$ came loose from the carrier, although not completely so that re-entrance could be carried out without misalignment at reinsertion during the next loading cycle. The test frequency was $7.5 \mathrm{~mm} / \mathrm{s}$ at the optimal stroke, resulting in a test frequency of $0.5 \mathrm{~Hz}$.

Graphs of the waveforms were captured every 36 cycles, starting at cycle 10 and ending at cycle 694 . On the load graphs, the point at which the BluePro ${ }^{\circledR}$ came loose from the carrier was detected since the load dropped to zero and stayed at zero for the rest of the upward trajectory. At this point the load needed to give a flat line at zero, also indicating that the load-channel had been calibrated to zero correctly.

Measurements were compared using a paired Student's t-Test (Statistica Release 6, StatSoft Inc. Tulsa, USA) at a significance level of 0.05 .
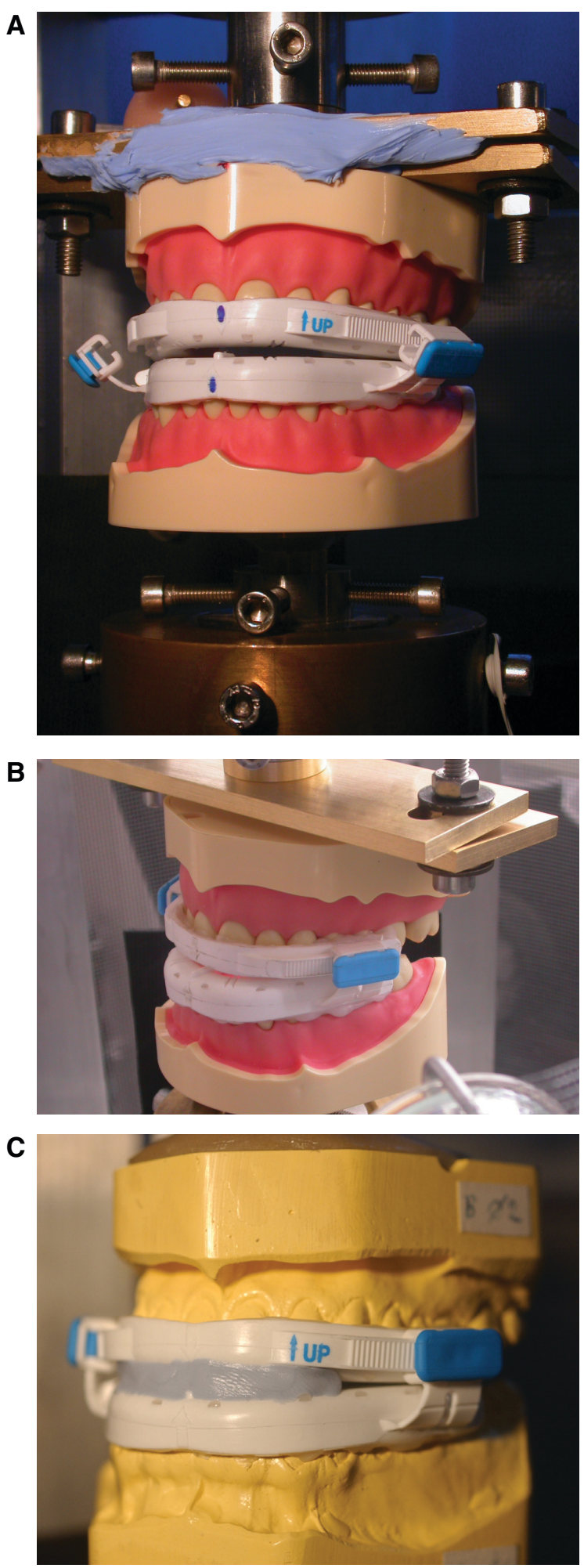

Figure 2. Casts and ANA-4 models used to test the retentive characteristics of the BluePro ${ }^{\circledR}$ device. (A) positioning of the BluePro ${ }^{\circledR}$ device on the ANA-4 carriers that are connected to the pistons of the servo-hydraulic fatigue machine, prior to tightening of the titration clips; (B) situation after tightening of the titration clips, joining the upper and lower part of the BluePro ${ }^{\circledR}$ device; (C) identical situation as in 'B' but on the epoxified gypsum carriers. 


\section{Results}

The removal forces $(\mathrm{N})$ expressed as a mean $( \pm \mathrm{SD})$ for both protrusion positions $(3 \mathrm{~mm}$ and $8 \mathrm{~mm})$ at the beginning and end of the tests revealing the effect of test duration are shown in Table 1: there was a slight but not significant decrease in retentive forces for the tests on the epoxified carriers, not to be found for the ANA-4 carriers. There were no statistical differences between the different test conditions and carriers as a function of the protrusion for the BluePro ${ }^{\circledR}$.

Inspection of the inner surfaces of the test samples revealed the presence of some wear particles and tear characteristics as shown in Figures $3 \mathrm{~A}$ and $3 \mathrm{~B}$.

Table 1. Retentive forces (N) with standard deviation (SD) at the start and end of the test cycles for both the epoxified casts and ANA-4 model supports.

\begin{tabular}{|l|l|l|l|l|l|}
\hline Protrusion & & \multicolumn{2}{|c|}{ Cast } & \multicolumn{2}{c|}{ ANA-4 } \\
\hline & & Cycle 10 & $\begin{array}{l}\text { Cycle } \\
\mathbf{6 9 4}\end{array}$ & Cycle 10 & $\begin{array}{l}\text { Cycle } \\
\mathbf{6 9 4}\end{array}$ \\
\hline $3 \mathrm{~mm}$ & Mean (N) & 26.6 & 24.2 & n/a & n/a \\
\hline & SD $(\mathrm{N})$ & 4.4 & 3.2 & n/a & n/a \\
\hline & n samples & 10 & 10 & n/a & n/a \\
\hline $\mathbf{8 m m}$ & Mean (N) & 25.7 & 24.3 & 27.4 & 27.4 \\
\hline & SD (N) & 3.4 & 2.9 & 5.9 & 6.6 \\
\hline & n samples & 10 & 10 & 10 & 10 \\
\hline
\end{tabular}

Dataset 1. Raw data of MRA-fatigue tests

http://dx.doi.org/10.5256/f1000research.6061.d43120

Detailed information on each data file can be found in the txt file provided

\section{Dataset 2. Data of retentive forces of mandibular advancement device BluePro ${ }^{\circledR}$}

59 Data Files

http://dx.doi.org/10.6084/m9.figshare.1306562

\section{Discussion}

The setup used in this study extends the experimental setup used previously ${ }^{8}$ by introducing a more standardized set of carriers with respect to tooth anatomy and shape of the tooth arcs. The results indicate that the measurements did not differ significantly between the two types of carriers, namely epoxified casts versus ANA-4 Frasaco models.

In OAm therapy for OSA it has not yet been defined to what extent variations in OAm design may affect clinical efficacy, side effects and patient compliance ${ }^{9}$. Design features may also dictate retention
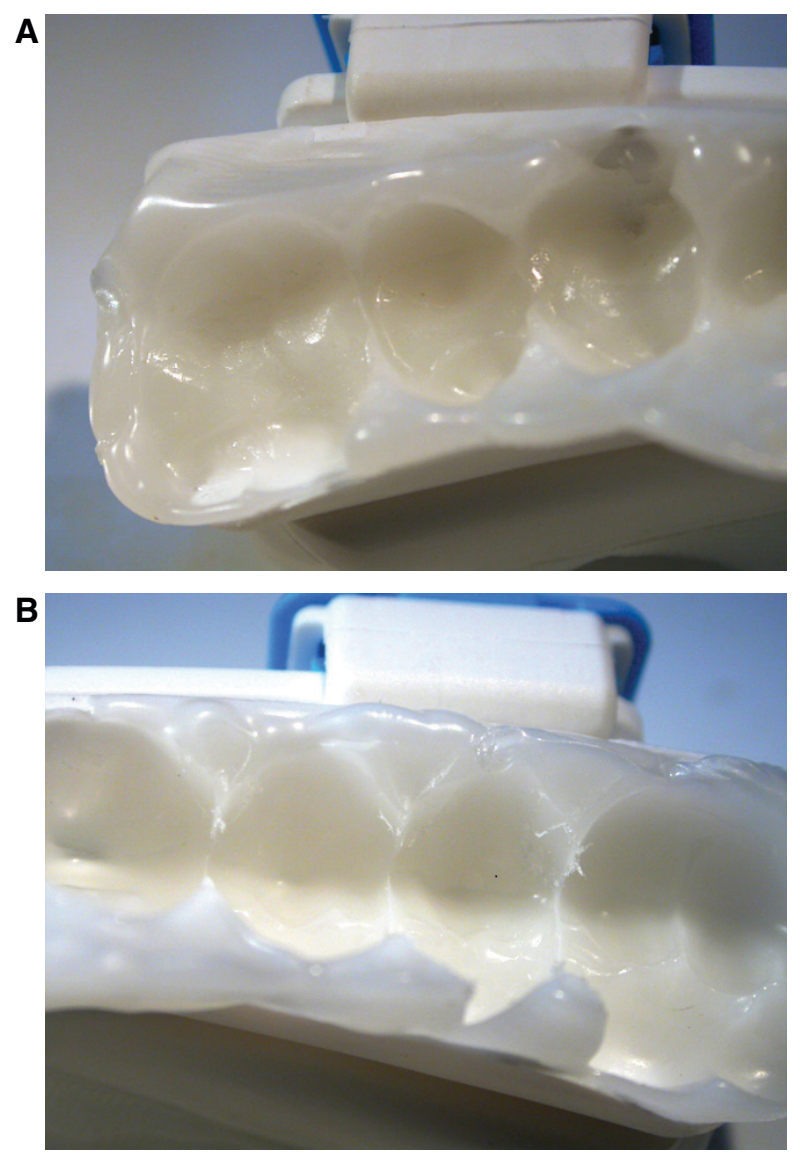

Figure 3. Wear particles and tear characteristics on the BluePro ${ }^{\circledR}$ device. (A) Almost none to (B) moderate signs of wear due to the repetitive loading, presenting itself under the form of small wear particles that are torn off the thermoplastic material as a consequence of the cyclic loading

of the OAm during sleep. Knowledge of such retentive characteristics is therefore essential for selection of an effective OAm for clinical use. It has previously been shown that it is feasible to evaluate in vitro retentive characteristics of monobloc types of $\mathrm{OAm}^{8}$ and the results of the present study indicate that a duo-bloc OAm can also be tested in a fixed protrusive position, thereby resembling the monobloc setup. The present findings also indicate that, compared to the previously published results, the experimental OAm currently tested at $8 \mathrm{~mm}$ protrusion showed retentive forces of $\sim 27 \mathrm{~N}$ as shown in Table 1. These findings further support the hypothesis 8 that introducing a soft thermoplastic body inside a more rigid tray improves retention of a thermoplastic type of OAm by improving rigidity and fit of the OAm. The present conclusion also supports recent findings with another prefabricated thermoplastic OAm that is also contained in a rigid shell ${ }^{7}$.

In the present study, the retention forces measured for the thermoplastic OAm did not change significantly over time (Table 1) and running-in wear and wear due to repetitive insertion and removal did not significantly influence the results. An increased amount of protrusion also did not cause higher retention forces (Table 1), 
indicating that its retentive capacities are independent of mandibular protrusion for the protrusive range studied. Additional research and clinical reports are needed to further study these effects.

Jaw opening force ${ }^{10}$ at an interincisal distance of about $10 \mathrm{~mm}$ range from $2 \mathrm{~N}$ to $9 \mathrm{~N}$ and increase thereafter to an average of $19.9 \pm 4.5 \mathrm{~N}$ at an interincisal distance of about $49 \mathrm{~mm}$. Looking at these values from the literature, it becomes clear that the thermoplastic OAm tested in the present in vitro study possesses sufficient retention forces to resist initial jaw opening as well as full mouth opening forces.

Often customizable thermoplastic OAms are less expensive than custom-made appliances in the dental technical lab. This additional element could broaden the indications for use of such devices. Since titration is now considered an inevitable prerequisite for obtaining success in OA treatment, better selection of appropriate patients for OAm therapy is essential. One approach to the selection of patients could be to provide the patient with a low-cost try-out device that is capable of reliable titration. Such a diagnostic tool would not only provide an evaluation of the effectiveness of an OAm in an at-home situation, but would also help check whether or not the patient is willing to use an OAm. It could also provide a temporary OAm while a custom-made OAm is being manufactured or repaired. Finally it could bring an OAm solution to certain resource-restricted healthcare settings in which custommade appliances are unaffordable. Although these indications seem promising, it should be noted that some elements regarding the design of an OAm and its effects are not yet known. This includes the bulkiness of the OAm and its effect on the position of the tongue for example.

\section{Data availability}

F1000Research: Dataset 1. Raw data of MRA-fatigue tests, 10.5256/f1000research.6061.d43120 ${ }^{11}$

Figshare: Data of retentive forces of mandibular advancement device BluePro ${ }^{\circledR}$. 10.6084/m9.figshare.1306562 ${ }^{12}$

\section{Competing interests}

The author is the promotor of a SomnoMed Research Grant. The author has no competing interest relating to BlueSom.

\section{Grant information}

BlueSom France provided funding for this project including the supply of BluePro ${ }^{\circledR}$ devices free of charge and payment for final manuscript editing by Newmed Publishing Services.
1. Dieltjens $\mathrm{M}$, Vanderveken $\mathrm{OM}$, Heyning $\mathrm{PH}$, et al:: Current opinions and clinical practice in the titration of oral appliances in the treatment of sleep-disordered breathing. Sleep Med Rev. 2012; 16(2): 177-85. PubMed Abstract | Publisher Full Text

2. de Almeida FR: Complexity and efficacy of mandibular advancement splints: understanding their mode of action. J Clin Sleep Med. 2011; 7(5): 447-8. PubMed Abstract | Publisher Full Text | Free Full Text

3. Lettieri CJ, Paolino N, Eliasson AH, et al:: Comparison of adjustable and fixed oral appliances for the treatment of obstructive sleep apnea. J Clin Sleep Med. 2011; 7(5): 439-45.

PubMed Abstract | Publisher Full Text | Free Full Text

4. Vanderveken OM, Devolder A, Marklund M, et al:: Comparison of a custom-made and a thermoplastic oral appliance for the treatment of mild sleep apnea. $A m J$ Respir Crit Care Med. 2008; 178(2): 197-202. PubMed Abstract | Publisher Full Text

5. Hoffstein V: Review of oral appliances for treatment of sleep-disordered breathing. Sleep Breath. 2007; 11(1): 1-22. PubMed Abstract | Publisher Full Text | Free Full Text

6. Tsuda H, Almeida FR, Masumi S, et al:: Side effects of boil and bite type oral appliance therapy in sleep apnea patients. Sleep Breath. 2010; 14(3): 227-32. PubMed Abstract | Publisher Full Text
7. Friedman M, Hamilton C, Samuelson CG, et al: Compliance and efficacy of titratable thermoplastic versus custom mandibular advancement devices. Otolaryngol Head Neck Surg. 2012; 147(2): 379-86. PubMed Abstract | Publisher Full Text

8. Vanderveken OM, Van de Heyning P, Braem MJ: Retention of mandibula advancement devices in the treatment of obstructive sleep apnea: an in vitro pilot study. Sleep Breath. 2014; 18(2): 313-8.

PubMed Abstract | Publisher Full Text | Free Full Text

9. Chan AS, Cistulli PA: Oral appliance treatment of obstructive sleep apnea: an update. Curr Opin Pulm Med. 2009; 15(6): 591-6.

PubMed Abstract | Publisher Full Text

10. Peck CC, Sooch AS, Hannam AG: Forces resisting jaw displacement in relaxed humans: a predominantly viscous phenomenon. J Oral Rehabil. 2002; 29(2): 151-60.

PubMed Abstract | Publisher Full Text

11. Spray I: Dataset 1 in "In vitro retention of a new thermoplastic titratable mandibular advancement device" F1000Research. 2015. Data Source

12. Spray I: Data of retentive forces of mandibular advancement device BluePro ${ }^{\circledR}$ Figshare. 2014 Data Source 


\section{Open Peer Review}

\section{Current Peer Review Status:}

\section{Version 1}

Reviewer Report 30 March 2015

https://doi.org/10.5256/f1000research.6489.r7832

(C) 2015 Cohen-Levy J. This is an open access peer review report distributed under the terms of the Creative Commons Attribution License, which permits unrestricted use, distribution, and reproduction in any medium, provided the original work is properly cited.

\section{Julia Cohen-Levy \\ Private Practice, Paris, France}

This is a well designed in-vitro study, supported with adequate experimental data and statistical analysis. Results are accurately presented and discussed, in a clear and concise manner. The conclusions are justified on the basis of the presented results.

Some minor revisions could be positively added to the manuscript, in the discussion part. The flaws of the 'in-vitro' study of the retention of a thermoplastic oral device should, in my opinion, be also discussed as the human mouth motion has very little to do with the testing machine, in relation to lateral movements of the mandible. The influence of saliva, and maybe of mouth temperature might have an influence on the material properties with time.

Competing Interests: No competing interests were disclosed.

\section{I confirm that I have read this submission and believe that I have an appropriate level of expertise to confirm that it is of an acceptable scientific standard.}

Reviewer Report 05 March 2015

\section{https://doi.org/10.5256/f1000research.6489.r7860}

(c) 2015 Quinnell T. This is an open access peer review report distributed under the terms of the Creative Commons Attribution License, which permits unrestricted use, distribution, and reproduction in any medium, provided the original work is properly cited.

\section{Timothy Quinnell}

Respiratory Support and Sleep Centre, Papworth Hospital NHS Foundation Trust, Papworth Everard, UK

The title accurately conveys the article's content. The abstract is a good summary of the work 
being reported. The author clearly describes the application of an in vitro method for measuring the retentive forces of mandibular devices, to a new titratable thermoplastic device. This novel method was recently reported by the author and colleagues (citation 8). It seems to be a useful in vitro tool for assessing the ability of mandibular devices to resist displacement by jaw opening - an important failing of many thermoplastic devices is poor oral retention. The technique naturally has its limitations, which have been discussed in detail in citation 8. It would be worth the author briefly referring to them in this article including: lack of lubrication; linear carrier movement vs. non-linear, 'least resistance' pathway of jaw opening in vivo; the use of carriers from only 1 patient. It is not clear how 730 cycles represents a year's use. If it refers to jaw opening at the end of the night to remove the device then should this not be 365 ? Nonetheless the data are credible and provide useful insight (alongside citation 8) into the varying in vivo retentive properties of different devices; and device durability; and further demonstrate a potentially straightforward means of in vitro OA testing. The protrusions used were clinically relevant. I agree with the author's conclusions, that the results suggest thermoplastic OAms (with adequate retentive properties) could have a role in: titration; temporary custom made device substitution; and longer term treatment. However I disagree that titration is now a prerequisite for successful OA treatment, because there is strong evidence that non-titratable devices do work (Thorax 2014;69:10 938-945); and the superiority of titration has not been proven by rigorous head-tohead trials. Finally, device effectiveness depends as much on comfort and tolerability (influencing adherence) as on oral retention.

Competing Interests: No competing interests were disclosed.

\section{I confirm that I have read this submission and believe that I have an appropriate level of expertise to confirm that it is of an acceptable scientific standard.}

The benefits of publishing with F1000Research:

- Your article is published within days, with no editorial bias

- You can publish traditional articles, null/negative results, case reports, data notes and more

- The peer review process is transparent and collaborative

- Your article is indexed in PubMed after passing peer review

- Dedicated customer support at every stage

For pre-submission enquiries, contact research@f1000.com

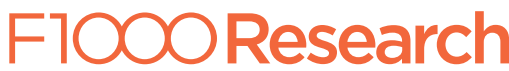

